\title{
Robust Multi-scale Non-rigid Registration of 3D Ultrasound Images
}

\author{
Ioannis Pratikakis, Christian Barillot, and Pierre Hellier \\ IRISA, INRIA-CNRS, ViSTA Project \\ Campus universitaire de Beaulieu, 35042 Rennes Cedex, France \\ i.pratikakis@ieee.org, cbarillo@irisa.fr, phellier@irisa.fr
}

\begin{abstract}
In this paper, we embed the minimization scheme of an automatic 3D non-rigid registration method in a multi-scale framework. The initial model formulation was expressed as a robust multiresolution and multigrid minimization scheme. At the finest level of the multiresolution pyramid, we introduce a focusing strategy from coarse-to-fine scales which leads to an improvement of the accuracy in the registration process. A focusing strategy has been tested for a linear and a non-linear scale-space. Results on 3D Ultrasound images are discussed.
\end{abstract}

\section{Introduction}

Non-rigid registration can be considered as a motion estimation problem which can be solved by minimizing an objective function. This function is the energy which usually consists of two terms. The first term represents the interaction between the unknown variables and the data while the second one explores some kind of prior information. In the context of dense motion field estimation, Mémin and Pérez [8] proposed a motion estimator which makes use of the optical flow constraint along with an associated smoothness regularizing prior. Both terms have been constructed with an outlier rejection mechanism, originated from robust statistics. For the minimization of their functional they use a multiresolution and multigrid scheme. The multiresolution part is dedicated to grasp large displacements while the multigrid approach is invoked for accelerating the estimation. Extension of this work to treat 3D data has been done by Hellier et al [5].

In this paper, we embed the above mentioned minimization scheme in a multiscale framework aiming to improve the estimates by making them less sensitive to noise of acquisition. In the same spirit, Weber and Malik [7] propose a model for multi-scale motion estimation. They convolve an image sequence with a set of linear, separable spatiotemporal filter kernels and apply a robust version of the total least squares on the filtered responses in a two step method. Niessen et al. 11] report a reconciliation of optical flow and scale-space theory. They compute both zeroth and first order optic flow at multiple spatial and temporal scales and they apply a scale selection criterion which attributes in each pixel the optic flow at the chosen scale. Alvarez et al. [1] present an interpretation of a classic optical flow method by Nagel and Enkelmann [10] as a tensor-driven 
anisotropic diffusion approach. They avoid convergence to irrelevant local minima by embedding their method in a linear scale-space framework.

Our work was motivated by the application of tissue deformation tracking during surgery using 3D ultrasound. The problem of registration (motion estimation) in ultrasound images has been treated by different researchers. Morcy and Von Ramm [9] investigate the implementation of a correlation search scheme to estimate the $3 \mathrm{D}$ motion vectors and demonstrate the advantages over $2 \mathrm{D}$ correlation search using the Sum Absolute Difference (SAD) as a similarity measure. Strintzis and Kokkinidis [14 introduce a maximum likelihood block matching technique which corresponds to an accurate statistical description of ultrasound images. In [16] an adaptive mesh has been proposed for non-rigid tissue motion estimation from ultrasound image sequences. A deformable blocking matching algorithm has been developed which takes into consideration both similarity measures and strain energy caused by mesh deformation. In [12], Pennec et al. disseminate results regarding 3D Ultrasound registration using the demon's algorithm and a straightforward minimization of the sum of square of intensity differences criterion.

Non-rigid registration of 3D Ultrasound images poses a significant challenge due to the following shortcomings: (i) Low SNR of ultrasound images which are characterized by Rayleigh-governed speckle noise and corrupted by Gaussiandistributed electronic noise; (ii) motion ambiguities which arise when there is insufficient representation of spatial information. This holds in regions of image saturation or specular reflection and in homogeneous regions of weak acoustic scatterers; (iii) Speckle decorrelation. Since speckle patterns result from the constructive and destructive interference of ultrasonic echoes from numerous subresolvable elements, nonuniform movement of these scatterers in the tissue volume can cause temporal decorrelation of the speckle patterns.

The algorithm which is presented in this paper is designed to overcome the above shortcomings and lead to an accurate registration.

The paper is organized as follows. In Section [2 we present in detail the multiresolution and multigrid optimization scheme. Section 3 describes the multiscale framework that the optimization scheme is embedded. Section 4 is dedicated to experimental results and conclusions are drawn in Section 5

\section{Primary Registration Model}

\subsection{Formulation of the Registration Problem}

In this work, the registration problem is considered as a motion estimation problem. The optical flow hypothesis, introduced by Horn et Schunck [6], leads then to the minimization of the following cost function:

$$
U(\boldsymbol{w} ; f)=\sum_{s \in S}\left[\boldsymbol{\nabla} f(s, t) \cdot \boldsymbol{w}_{s}+f_{t}(s, t)\right]^{2}+\alpha \sum_{<s, r>\in \mathcal{C}}\left\|\boldsymbol{w}_{s}-\boldsymbol{w}_{r}\right\|^{2},
$$

where $s$ is a voxel of the volume, $t$ is the temporal index of the volumes, $f$ is the luminance function, $\boldsymbol{w}$ is the expected $3 D$ displacement field, $S$ is the voxel 
lattice, $\mathcal{C}$ is the set of neighboring pairs and $\alpha$ controls the balance between the two energy terms. The first term is the first order Taylor-expansion of the luminance conservation equation and represents the interaction between the field and the data, whereas the second term expresses the smoothness constraint.

Shortcomings of this formulation are well-known:

(a) The optical flow constraint (OFC) is not valid in case of large displacements because of the linearization.

(b) The OFC might not be valid everywhere, because of the noise, the intensity non-uniformity, and occlusions.

(c) The "real" field probably contains discontinuities that might not be preserved.

To cope with (b) and (c) limitations, the quadratic cost has been replaced by robust functions. To face problem (a), a multiresolution and multigrid strategy has been designed.

\subsection{Robust Estimators}

Cost function (11) does not make any difference between relevant data and inconsistent data, and it is sensitive to noise. Therefore, robust M-estimators have been introduced in the formulation [2]. An M-estimator is a function $\rho$ that is increasing on $\mathbb{R}^{+}$, such that (i) $\phi(u) \triangleq \rho(\sqrt{u})$ is strictly concave on $\mathbb{R}^{+}$and (ii) $\lim _{x \rightarrow \infty} \rho^{\prime}(x)<\infty$. The main benefit of robust M-estimators is the semiquadratic formulation that can be deduced from (i):

$$
\exists \psi \in C^{1}([0, M], \mathbb{R}): \forall u, \rho(u)=\min _{z \in[0, M]}\left(z u^{2}+\psi(z)\right)
$$

Two robust estimators have therefore been introduced: the first one on the data term $\left(\rho_{1}\right)$ and the second one on the regularization term $\left(\rho_{2}\right)$. According to (2), the minimization of the cost function (1) is equivalent to the minimization of the augmented function, noted $\stackrel{\star}{U}$ :

$$
\begin{gathered}
\stackrel{\star}{U}(\boldsymbol{w} ; f)=\sum_{s \in S} \delta_{s}\left(\nabla f(s, t) \cdot \boldsymbol{w}_{s}+f_{t}(s, t)\right)^{2}+\psi_{1}\left(\delta_{s}\right) \\
+\alpha \sum_{<s, r>\in \mathcal{C}} \beta_{s r}\left(\left\|\boldsymbol{w}_{s}-\boldsymbol{w}_{r}\right\|\right)^{2}+\psi_{2}\left(\beta_{s r}\right),
\end{gathered}
$$

where $\delta_{s}$ and $\beta_{s r}$ are auxiliary variables acting as "weights". This cost function has the advantage to be quadratic with respect to $\boldsymbol{w}$. Furthermore, when the adequation of a data with the model is not correct, its contribution gets lower as the associated weight $\delta_{s}$ decreases $\left(\delta_{s}=\phi_{1}^{\prime}\left(\left[\boldsymbol{\nabla} f(s, t) \cdot \boldsymbol{w}_{s}+f_{t}\right]^{2}\right)\right.$, and function $\phi^{\prime}$ decreases), making this formulation more robust. 


\subsection{Multiresolution and Multigrid Minimization}

In order to cope with large displacements, a classical incremental multiresolution procedure has been developed. A pyramid of volumes $\left\{f^{k}\right\}$ is constructed by successive Gaussian smoothing and subsampling. At the coarsest level, the linearization of the conservation equation can be hopefully used. For the next resolution levels, only an increment $\mathrm{d} \boldsymbol{w}^{k}$ is estimated to refine estimate $\hat{\boldsymbol{w}}^{k}$, obtained from the previous level (Equation 4).

$$
\begin{aligned}
& \stackrel{\star}{U^{k}}\left(\mathrm{~d} \boldsymbol{w}^{k} ; f^{k}, \hat{\boldsymbol{w}}^{k}\right)=\sum_{s \in S^{k}} \delta_{s}^{k}\left(\boldsymbol{\nabla} f^{k}\left(s+\hat{\boldsymbol{w}}_{s}^{k}, t_{2}\right) \mathrm{d} \boldsymbol{w}_{s}^{k}+f^{k}\left(s+\hat{\boldsymbol{w}}_{s}^{k}, t_{2}\right)-f^{k}\left(s, t_{1}\right)\right)^{2} \\
& +\psi_{1}\left(\delta_{s}^{k}\right)+\alpha \sum_{<s, r>\in \mathcal{C}^{k}} \beta_{s r}^{k}\left(\left\|\left(\hat{\boldsymbol{w}}_{s}^{k}+\mathrm{d} \boldsymbol{w}_{s}^{k}\right)-\left(\hat{w}_{r}^{k}+\mathrm{d} \boldsymbol{w}_{r}^{k}\right)\right\|\right)^{2}+\psi_{2}\left(\beta_{s r}^{k}\right)
\end{aligned}
$$

Furthermore, at each level of resolution, a multigrid minimization based on successive partitions of the initial volume is achieved (see Fig. 1). For each cube of a given grid level $\ell$ (partition of cubes), a 12-parametric increment field is estimated. The result over the grid level is a rough estimate of the desired solution, and it is used to initialize the next grid level. This hierarchical minimization strategy improves the quality and the convergence rate.

The partition at the coarsest grid level is initialized with a binary segmentation mask of the structure of interest (template). The octree partition which is thus defined is anatomically relevant. When we change grid level, each cube is adaptively divided. The criterion of subdivision may be either the measure of the way that model fits the data, or a prior knowledge such as the presence of an important anatomical structure where estimation must be accurate. Consequently, we can distinguish between the regions of interest where the estimation must be precise and the other regions where computation efforts are useless.

\section{Embedded Multi-scale Framework}

The multigrid scheme which has already been described is bound to a good initialization of the flow. To improve the quality of the initial estimates we propose to incorporate the scale of image measurements by exploring the scalespace of the data-derived information. Specifically, since we deal with the optical flow constraint we experiment with two scale-spaces which are characterized by the luminance conserving principle. These are the linear scale-space [15] and the one which is constructed by the regularized version [4] of Perona-Malik (P\&M) algorithm [13]. Let $f_{\tau}^{0}$ be the luminance of a voxel at the finest spatial resolution which has been diffused at the scale quantization level $\tau$. Then, a linear scalespace is denoted as:

$$
f_{\tau}=f_{o} * G_{\sigma}
$$

where $*$ denotes convolution, $f_{o}$ is the original image and $G_{\sigma}$ is the Gaussian kernel for standard deviation $\sigma$. 


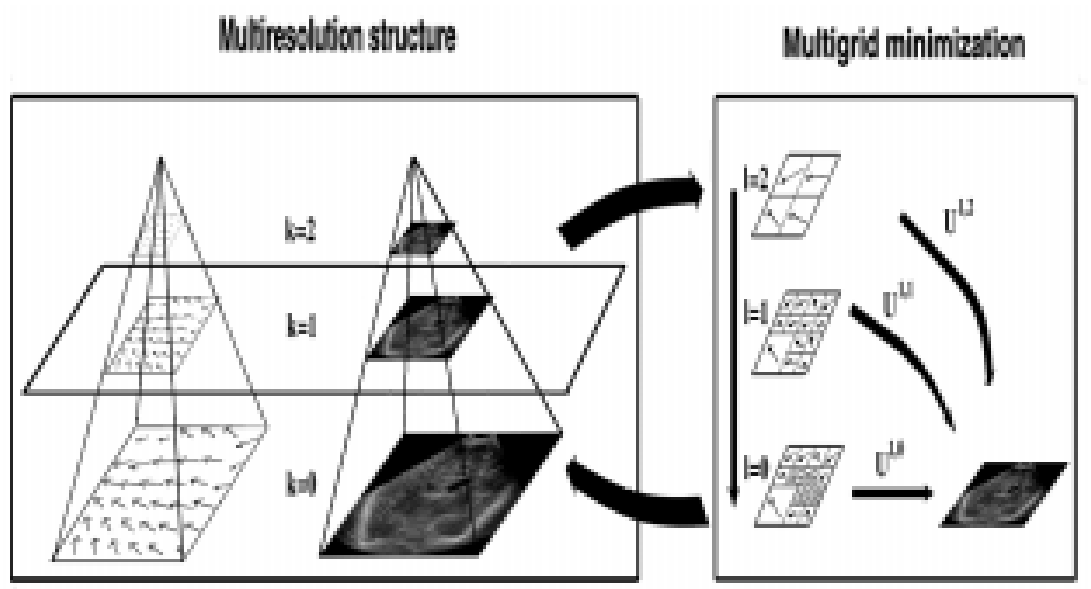

Fig. 1. Example of multiresolution/multigrid minimization. For each resolution level (on the left), a multigrid strategy (on the right) is performed. For clarity reasons, this is a $2 D$ illustration of our $3 D$ algorithm.

If no scale is preferred, the natural way to travel through a linear multi-scale can be realized via a sampling which should follow a linear and dimensionless scale parameter $\delta \lambda$ which is related to $\sigma$ by :

$$
\sigma_{\tau}=e^{\lambda_{0}+\tau \delta \lambda}
$$

where $\tau$ denotes the scale quantization levels.

The regularized $\mathrm{P} \& \mathrm{M}$ scale-space in its discretized form is denoted as:

$$
f_{\tau}=f_{\tau-1}+\lambda \sum c_{i}\left(G_{\sigma} * \Delta_{i} f\right)
$$

where $i \in\{N, S, E, W, F, B\}$ and $N, S, E, W, F, B$ denote Northern, Southern, Eastern, Western, Forward and Backward neighbor respectively.

$$
c_{i}=g\left\|G_{\sigma} * \Delta_{i} f\right\|
$$

$c_{i}$ is a decreasing function of the image gradient that has been determined at a scale $\sigma$ to compensate for noise and to assure well-posedness of the diffusion equation. $\Delta_{i} f=f_{i}-f_{\star}$ where $f_{\star}$ denotes the central pixel in a 3 -dimensional mask with 6-neighbor connectivity.

$$
\begin{gathered}
g\left\|\Delta_{i} f\right\|=e^{-\left(\frac{\Delta_{i}{ }^{2}}{k}\right)} \\
g\left\|\Delta_{i} f\right\|=\frac{1}{\left(1+\frac{\Delta_{i} f^{2}}{k}\right)}
\end{gathered}
$$

where $k$ is a contrast parameter and can be interpreted as a threshold, which determines whether a gradient is significant or not. 
For non-linear diffusion schemes there is no global scale parameter because they adapt the diffusion locally. However, we may synchronize their scale parameter with the one of linear diffusion. This holds due to the fact that the scalar diffusivity $c_{i}$ in Equation 8 is constructed such that $\|c\| \leq 1$. Therefore, an upper bound is derived for the nonlinear schemes which permits us to recall the relation between the evolution parameter and the standard deviation of the Gaussian $\tau_{n}=(1 / 2) \sigma_{n}^{2}$ for the creation of the regularized P\&M scale quantization space.

The construction of any of the above scale-spaces leads to a stack of volumes $\left\{f_{\tau}^{0}\right\}$ which is the source of the data measurements for every successive quantization scale during a coarse-to-fine parameter estimation. This can be explained by Equation 11

$$
\begin{aligned}
& \stackrel{\star}{U_{\tau}^{0}}\left(\mathrm{~d} \boldsymbol{w}^{0} ; f_{\tau}^{0}, \hat{\boldsymbol{w}}^{0}\right)=\sum_{s \in S^{0}} \delta_{s}^{0}\left(\nabla f_{\tau}^{0}\left(s+\hat{\boldsymbol{w}}_{s}^{0}, t_{2}\right) \cdot \mathrm{d} \boldsymbol{w}_{s}^{0}+f_{\tau}^{0}\left(s+\hat{\boldsymbol{w}}_{s}^{0}, t_{2}\right)-f_{\tau}^{0}\left(s, t_{1}\right)\right)^{2} \\
& +\psi_{1}\left(\delta_{s}^{0}\right)+\alpha \sum_{<s, r>\in \mathcal{C}^{0}} \beta_{s r}^{0}\left(\left\|\left(\hat{\boldsymbol{w}}_{s}^{0}+\mathrm{d} \boldsymbol{w}_{s}^{0}\right)-\left(\hat{w}_{r}^{0}+\mathrm{d} \boldsymbol{w}_{r}^{0}\right)\right\|\right)^{2}+\psi_{2}\left(\beta_{s r}^{0}\right),
\end{aligned}
$$

$f_{\tau}^{0}$ denotes the data measurement at the finest pyramid resolution and the $\tau$ scale quantization level.

Our goal is the estimation of parameter $\hat{\boldsymbol{w}}^{0}$ which is refined at each quantization scale by only an increment $\mathrm{d} \boldsymbol{w}_{s}^{0}$. Minimization remains in the same multigrid fashion.

\section{Experimental Results}

We have already mentioned in Section 1 that our efforts were motivated by the application of tissue deformation tracking which can result in brain shift correction. In view of this, we have conducted a number of experiments using an original 3D Ultrasound image $(256 \times 256 \times 128)$ of the brain of an 8 months old baby and its deformed counterpart. The acquired original volume is the result of an examination through the fontanella. In the ideal case, the accuracy of our algorithm in registering volumes should be tested in a situation that the actual motion should be known. Due to the difficulty to produce known non-rigid motion fields in biological tissues we have chosen to simulate this phenomenon. We have created an artificially deformed volume by using a Thin Plate Spine deformation [3]. Although this approach produces a global smooth deformation, we were very careful in the distribution of the point landmarks over the whole volume to cope with local deformations. The produced deformed volume and velocity field can be seen in Figure 2(b) and Figure 2(c), respectively.

In our experimental work we strived towards an overall comparison between the primary non-rigid registration model of Section 2 and the model with an embedded scale-space framework of Section 3. Our evaluation is both qualitative and quantitative. As a qualitative measure we have chosen to use the difference image between the original volume and the reconstructed one. All of the registration models produced difference images without significant differences, 
implying a visually correct registration (Figure 3(b)). For the sake of comparison we provide you the difference image between the original volume and the deformed one in Figure [3(a). The difference image in Figure 33(b) has come out after the application of the algorithm which uses the embedded regularized P\&M scale-space.

For a quantitative evaluation we have considered the following measures : (i)Mean square error (MSE); (ii) the average angular error between correct $\overrightarrow{v_{c}}$ and estimated $\overrightarrow{v_{e}}$ velocity : $\psi=\arccos \left(\overrightarrow{v_{c}} \cdot \overrightarrow{v_{e}}\right)$ along with (iii) its standard deviation. Table 1 demonstrates the improvement in velocity estimation which

Table 1. Quantitative Comparison Measures.

\begin{tabular}{|c|c|c|c|}
\hline & MSE & Mean angular error & Std deviation \\
\hline Without multi-scale framework & 10.2772 & $14.112656^{\circ}$ & $24.254787^{\circ}$ \\
Embedded Linear scale-space & 9.73472 & $13.878700^{\circ}$ & $23.987515^{\circ}$ \\
Embedded Regularized P\&M scale-space & 9.6945 & $13.791579^{\circ}$ & $23.959972^{\circ}$ \\
\hline
\end{tabular}

has been achieved for all three above measures in the case of the embedded scale-space framework for both the linear and the regularized P\&M case. The latter one has a slightly better behavior than the linear one.

Our basic argumentation for the advantageous use of a multi-scale framework was that it can lead to improvement in quality of the initial estimates at the multigrid optimization scheme which subsequently will improve the quality of the final estimates. A verification of this is presented in Figure 3(c) which shows in terms of MSE the improvement that occurs during successive multigrid levels at the finest spatial resolution for all the three examined cases. We may observe that in the case of the absence of a multi-scale framework we get an initial estimate with an MSE equals to 15.1756 while in the case of linear scale-space we get an initial estimate with an MSE equals to 11.9146 and in the case of regularized $\mathrm{P} \& \mathrm{M}$ scale-space we get an initial estimate with an MSE equals to 12.1082. The higher quality of the initial estimates was preserved till the final stage at the multigrid optimization scheme.

\section{Conclusions}

In this paper, we propose a methodology which embeds a multi-scale framework in a multiresolution and multigrid optimization scheme that can lead to a successful non-rigid registration of 3D Ultrasound images. It grasps its power from three fundamental features which operate as the remedy in the basic shortcomings of ultrasound images. Its multigrid nature responds to motion ambiguities in the case of insufficient representation of spatial information, its estimate smoothness functional term can fight the speckle decorrelation which characterizes ultrasound while low SNR can be less disastrous for the estimates in the case of embedding a multi-scale framework. 
In our last word will keep on defending the use of a multi-scale framework but it will not provide any definite clue about the superiority of either the linear or a non-linear scale-space. We opt on experimenting with more non-linear scalespaces in order to reach a definite and generalized conclusion.

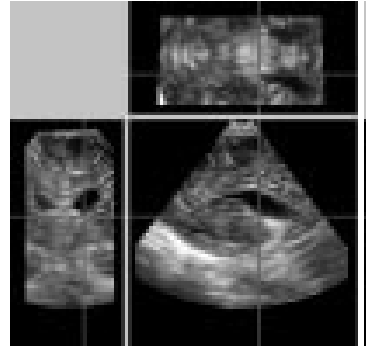

(a)

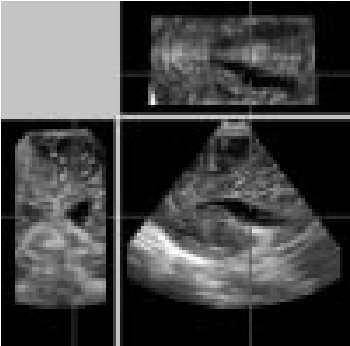

(b)

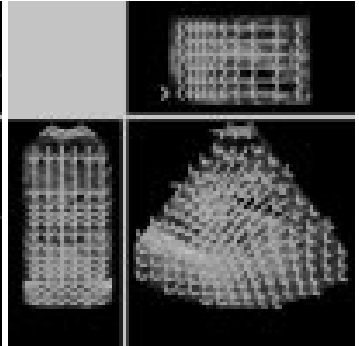

(c)

Fig. 2. (a) Preoperative 3D Ultrasound; (b) Simulated intraoperative (Deformed) 3D Ultrasound; (c) The artificial deformation field.

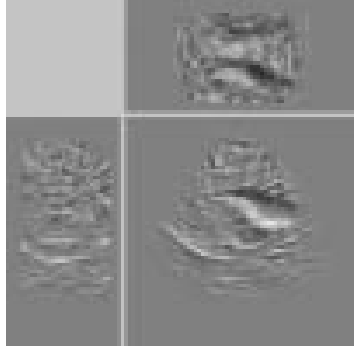

(a)

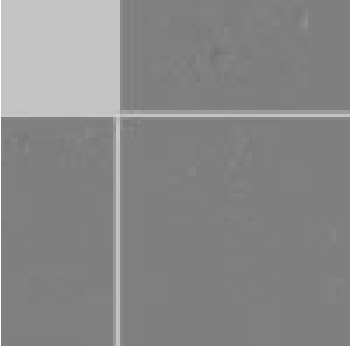

(b)

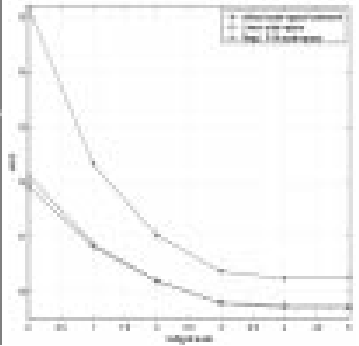

(c)

Fig. 3. (a) Difference between the original and the deformed volume; (b) Difference between the original and the reconstructed volume; (c) MSE improvement wrt to multigrid levels at the finest spatial resolution.

Acknowledgments. The 3D US image of the baby was provided by Prof. Auer and his colleagues at ISM (Austria) in the framework of the EC-funded ROBOSCOPE project (HC 4018), a collaboration between the Fraunhofer Institute (Germany), Fokker Control System (Netherlands), Imperial College (UK), INRIA (France), ISM-Salzburg and Kretz Technik (Austria). This work has been granted by INRIA within the Cooperative Action Framework which involved the Epidaure and ISA projects of INRIA. Finally, we are grateful to Xavier Pennec and Nicholas Ayache for their support in this work. 


\section{References}

1. L. Alvarez, J. Weickert, and J. Sanchez. A scale-space approach to nonlocal optical flow calculations. In Scale-Space '99, pages 235-246, 1999.

2. M. Black and A. Rangarajan. On the unification of line processes, outlier rejection and robust statistics with applications in early vision. International Journal of Computer Vision, 19(1):57-91, 1996.

3. F.L. Bookstein. Principal warps: Thin-plate slines and the decomposition of defomations. IEEE Transactions on Pattern Analysis and Machine Intelligence, 11(6):567-585, 1989.

4. F. Catté, P.L. Lions, J.M. Morel, and T. Coll. Image selective smoothing and edge detection by nonlinear diffusion. SIAM Journal on Numerical analysis, 29:182-193, 1992.

5. P. Hellier, C. Barillot, E. Mémin, and P. Pérez. An energy-based framework for dense $3 \mathrm{D}$ registration of volumetric brain images. In IEEE Conf. on Computer Vision and Pattern Recognition (CVPR), volume II, pages 270-275, Hilton Head Island, South Carolina, USA, June 2000.

6. B. Horn and B. Schunck. Determining optical flow. Artificial Intelligence, 17:185203, August 1981.

7. J.Weber and J. Malik. Robust computation of optical flow in a multi-scale differential framework. International Journal of Computer Vision, 14:67-81, 1995.

8. E. Mémin and P. Pérez. Dense estimation and object-based segmentation of the optical flow with robust techniques. IEEE Transactions on Image Processing, 7(5):703-719, 1998.

9. A. Morsy and O. VonRamm. 3D ultrasound tissue motion tracking using correlation search. Ultrasonic Imaging, 20:151-159, 1998.

10. H.H. Nagel and W. Enkelmann. An investigation of smoothness constraints for the estimation of displacement vector fields from image sequences. IEEE Transactions on Pattern Analysis and Machine Intelligence, 8:565-593, 1986.

11. W.J. Niessen, J.S. Duncan, M. Nielsen, L.M.J. Florack, ter Haar Romeny B.M, and M.A. Viergever. A multiscale approach to image sequence analysis. Computer Vision and Image Understanding, 65(2):259-268, 1997.

12. X. Pennec, P. Cachier, and N. Ayache. Understanding the "demon's algorithm": 3D non-rigid registration by gradient descent. In MICCAI, pages 597-605, September 1999.

13. P. Perona and J. Malik. Scale-space and edge detection using anisotropic diffusion. IEEE Transactions on Pattern Analysis and Machine Intelligence, 12(7):629-639, 1990.

14. M. Strintzis and I. Kokkinidis. Maximum likelihood motion estimation in ultrasound image sequences. IEEE Signal Processing Letters, 4(6):156-157, 1997.

15. A.P. Witkin. Scale-space filtering. In International Joint Conference on Artificial intelligence, pages 1019-1023, Karlsruhe, W. Germany, 1983.

16. F. Yeung, S. Levinson, D. Fu, and K. Parker. Feature-adaptive motion tracking of ultrasound image sequences using a deformable mesh. IEEE Transactions on Medical Imaging, 17(6):945-956, 1998. 